\title{
Ubiquitin-conjugating enzyme UbcH10 promotes gastric cancer growth and is a potential biomarker for gastric cancer
}

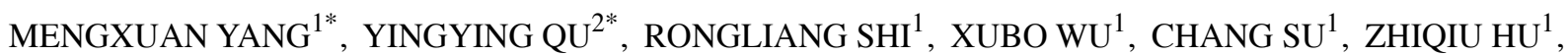 \\ QIMENG CHANG ${ }^{1}$, SHAOQUN LIU ${ }^{1}$, GAOFENG PAN ${ }^{1}$, MING LEI $^{1}$, FUBO XIE $^{2,4}$, SHIWEI TU ${ }^{2}$, \\ WEIKANG TAO ${ }^{2}$, $\mathrm{HE} \mathrm{ZHOU}^{2,3}, \mathrm{GANG} \mathrm{HU}^{2-4}$ and ZIPING ZHANG ${ }^{1}$ \\ ${ }^{1}$ Minhang Hospital, Fudan University, Shanghai 201199; ${ }^{2}$ Shanghai ChemPartner Co., Ltd., \\ Shanghai 201203; ${ }^{3}$ Shanghai Engineering Research Center of Pharmaceutical Translation, \\ Shanghai 201203; ${ }^{4}$ ClinicalExplorer (Shanghai) Co., Ltd., Shanghai 201203, P.R. China
}

Received February 1, 2016; Accepted March 9, 2016

DOI: 10.3892/or.2016.4906

\begin{abstract}
Gastric cancer is a fatal disease and the availability of early diagnostic methods is limited. There is an urgent need to identify effective targets for early diagnosis and therapeutics. UbcH10 is a ubiquitin-conjugating enzyme with high expression in various types of cancers. In the present study, several gastric tumor cell lines with high or low expression of UbcH10 were exploited to study the role of $\mathrm{UbcH} 10$ in gastric cancer. Knockdown of UbcH10 expression using siRNA in gastric cancer cell lines with high expression of UbcH10 resulted in reduced proliferation, increased cisplatin-induced apoptosis and reduced serum-induced ERK, Akt and p38 phosphorylation signaling. In agreement, overexpression of $\mathrm{UbcH} 10$ in gastric cancer cell lines with low expression of UbcH10 led to enhanced cell proliferation and resistance to cisplatin-induced apoptosis. Most importantly, IHC analyses showed that the UbcH10 protein was expressed at a high level in most patient gastric cancer tissues, but was absent in adjacent mesenchyme tissues. These data suggest that $\mathrm{UbcH} 10$ may promote gastric cancer growth and can serve as a biomarker for diagnosis or as a target for novel therapeutics in gastric cancer.
\end{abstract}

Correspondence to: Dr Gang Hu, Shanghai ChemPartner Co., Ltd., Building 6, R202, 998 Halei Road, Shanghai 201203, P.R. China

E-mail: ghu@chempartner.cn

Dr Ziping Zhang, Minhang Hospital, Fudan University, 170 XinSong Lu, Shanghai 201199, P.R. China

E-mail: zzpmd68@live.cn

*Contributed equally

Key words: UbcH10, gastric cancer, biomarker, tumor growth

\section{Introduction}

Gastric cancer is a multifactorial and fatal disease, and its overall mortality ranked third among cancer-related deaths worldwide in 2012 (1). The incidence and mortality rate of gastric cancer vary geographically, with the highest in East Asia, including China and Japan (2,3). Currently, surgical resection along with chemoradiation are the standard therapeutic procedures $(4,5)$. More research efforts to identify molecular biomarkers can facilitate early diagnosis or new drug development for gastric cancers. Several critical genes have been discovered to correlate with different types of gastric cancers (intestinal and diffuse types) and have been suggested as potential prognostic biomarkers in previous reviews (6-9).

Ubiquitin-dependent proteolysis is a significance physiological process involved in differentiation, signal transduction and the cell cycle $(10,11)$. Ubiquitin-activating enzyme (E1), ubiquitin-conjugating enzyme (E2) and ubiquitin ligase (E3) are the three different enzymes involved in this system. UbcH10 belongs to the E2 gene family coding for a protein of $19.6 \mathrm{kDa}$ and plays an important role in the destruction of mitotic cyclins and cell cycle progression (12-14).

Pulmonary tumors are among the neoplasms with reportedly high levels of $\mathrm{UbcH} 10$ and $\mathrm{UbcH} 10$ has been shown to play an important role in tumor progression (15). UbcH10 was found to be a useful target for diagnosis and therapy in a subsequent study (16). UbcH10 also presents a high expression level in anaplastic thyroid carcinomas (17), endometrial malignant neoplasms (18), nasopharyngeal carcinoma (19), liver cancer (20), breast (21) and colon carcinoma $(22,23)$, and brain metastasis (24). High expresssion of $\mathrm{UbcH} 10$ is correlated with cell invasion and tumor node metastasis (TNM) stage in some of the above-mentioned cancer types. Furthermore, knockdown of UbcH10 expression by RNA interference significantly reduced the proliferation of cancer cells $(17,25)$ and enhanced cell apoptosis in vitro (26).

To date, there is no study concerning UbcH10 and gastric carcinoma. To investigate this, two gastric cancer cell lines with a high UbcH10 expression level, KATO III and SGC-7901, 
were selected for gene knockdown experiments. UbcH10 was also transfected into NCI-N87 and HS-746T, two cell lines with low $\mathrm{UbcH} 10$ expression and low multiplication capacity. Cell proliferation, apoptosis and cell signal transductions were examined in these cell lines.

To examine the clinical relevance of the findings, paraffinembedded gastric carcinoma tissue samples from 59 patients were analyzed by immunohistochemistry.

\section{Materials and methods}

Clinical samples. Tumor samples from 59 cases of gastric carcinoma were obtained from the Minhang Central Hospital, (Shanghai, China) from 2012 to October 2014. The procedure was performed with patient informed consent and in accordance with the Helsinki Declaration and upon the approval of The Institutional Review Board of Minhang Hospital.

Cell lines. The human gastric carcinoma cell lines used in the present study were SGC-7901, AGS, NCI-N87, HS 746T, MKN-45, KATO III, NCI-SNU-1, SNU-5 and SNU-16 and were purchased from the American Type Culture Collection (ATCC; Manassas, VA, USA). These cell lines were cultured in Dulbecco's modified Eagle's medium (DMEM) containing $10 \%$ fetal calf serum, glutamine and ampicillin/streptomycin (all from Gibco Laboratories, Carlsbad, CA, USA) in a 5\% $\mathrm{CO}_{2}$ atmosphere. GAX023, GAXC031 and GAX066 are primary tumor cell lines established from PDX models (PDX-PDC; patient-derived xenograft, patient-derived cell line) at Shanghai ChemPartner as previously described (27-29).

Reagents. The anti-UbcH10 (A-650) antibody was purchased from Boston Biochem (Boston, MA, USA). Anti-ERK1/2 (\#4695), anti-phospho-ERK1/2 (T202/Y204, \#4370), anti-p38 MAPK (D13E1, \#8690), anti-phospho-p38 MAPK (Thr180/ Tyr182, \#9211), anti-Akt (\#9272), anti-phospho-Akt (Ser473, \#9271), anti-actin (13E5, \#4970) and anti-GAPDH (14C10) antibodies were purchased from Cell Signaling Technology (Danvers, MA, USA). Annexin V-FITC Apoptosis Detection Kit I (cat. 556547) was obtained from BD Pharmingen (San Diego, CA, USA). Blocking buffer (PBS), goat anti-mouse (IRDye800CW 926-32210) and goat anti-rabbit (IRDye800CW 926-32211) were purchased from Odyssey. Hoechst 33258 (\#861405) and MTT reagents (M2128) were purchased from Sigma-Aldrich (St. Louis, MO, USA).

Plasmid construction. The CDS sequences (121-660) of UBE2C transcript variant 1 mRNA (GeneBank NM_007019) were chemically synthesized and cloned into mammalian cell expression vector pLVX-UBE2C-IRES-eGFP.

Transfection of small interfering RNA. Gastric carcinoma cells were transfected using Lipofectamine 2000 transfection reagent (Invitrogen, Carlsbad, CA, USA) according to the manufacturer's instructions.

siRNA directed against the $\mathrm{UbcH} 10$ transcript with the sequence: 5'-CCUGCAAGAAACCUACUCAdTdT3' antisense, and a scrambled siRNA were obtained from GenePharma (Shanghai, China). In vitro transfections were performed using $0.8 \mu \mathrm{g}(54 \mathrm{pM})$ siRNA in 24 -well plates using
Lipofectamine 2000 transfection reagent $\left(1 \times 10^{5}\right.$ cells/well) following the manufacturer's protocol. Transfection of siRNA was performed at least 5 times for each cell line. Each experiment was repeated at least twice. Non-specific siRNAs (scrambled siRNAs) were used as negative controls.

Western blot assay. Cells were lysed in T-PER tissue lysis buffer containing protease inhibitor (both from Thermo Fisher Scientific, Waltham, MA, USA), and phosphatase inhibitor cocktail (Sigma, St. Louis, MO, USA). Twenty micrograms of protein/sample was loaded on 4-12\% SDS-PAGE gels (Life Technologies, Carlsbad, CA, USA). Proteins were separated and transferred onto a nitrocellulose membrane. Membranes were incubated with blocking buffer (LI-COR, Inc., Lincoln, NE, USA) and incubated with primary antibodies overnight, followed by the Alexa Fluor 680-conjugated secondary antibody (Invitrogen). Blots were scanned on the Odyssey system (LI-COR, Inc.). Anti-p-ERK1/2 (Thr202/Tyr204), anti-ERK1/2, anti-Akt, anti-p-Akt (Ser473), anti-p38, anti-pp38 and anti-cyclin D1 antibodies were purchased from Cell Signaling Technology. Anti-actin (Cell Signaling Technology) or anti-GAPDH antibody (Epitomics, Burlingame, CA, USA) was used as a loading control.

MTT assay. 3-(4,5-Dimethylthiazol-2-yl)-2,5-diphenyltetrazolium bromide (MTT) assay was performed to study the proliferation of the tumor cells. Cells were plated in 96-well plates at a density of $10^{4}$ cells/well to attach for $24 \mathrm{~h}$ and transfected with $50 \mathrm{nM}$ different siRNAs. At $0,24,48$ and $72 \mathrm{~h}$ after treatment, $10 \mu \mathrm{l}$ of MTT reagent ( $5 \mathrm{mg} / \mathrm{ml}$; Sigma) was added to each well. After a 4-h incubation at $37^{\circ} \mathrm{C}$, the supernatant was gently aspirated and formazan crystals were dissolved in $100 \mu \mathrm{l}$ of dimethyl sulfoxide (DMSO). The absorbance of each well was measured by an ELISA plate-reader (Bio-Rad, Hercules, CA, USA) at a wavelength of $570 \mathrm{~nm}$.

Cell cycle and apoptosis analysis. The cell cycle or apoptosis distribution was analyzed by flow cytometry. Cells were seeded into a 6-well plate, followed by transfection of siRNA or overexpression plasmids, and cultivation was maintained for $36 \mathrm{~h}$. For cell cycle analyses, the harvested cells were washed with PBS and fixed with ice-cold $70 \%$ ethanol at $4^{\circ} \mathrm{C}$ overnight. The fixed cells were resuspended in PBS (containing $0.2 \mathrm{mg} / \mathrm{ml}$ RNAse and $10 \mathrm{mM}$ PI) and incubated in the dark for $30 \mathrm{~min}$ at $4^{\circ} \mathrm{C}$.

For cell apoptosis analyses, the cells were harvested and washed with PBS and stained with Annexin V and PI staining (Annexin V-FITC Apoptosis Detection Kit; BD Biosciences, San Jose, CA, USA). A FACSCalibur flow cytometer (Becton-Dickinson, San Jose, CA, USA) was used for data acquisition and FlowJo (TreeStar, Palo Alto, CA, USA) was used for data analysis.

Immunohistochemistry. Protein cellular distribution of $\mathrm{UbcH} 10$ was assessed by immunohistochemical analysis. Formalin-fixed, paraffin-embedded, 6-mm tissue sections were deparaffined. Antigen retrieval was performed by water heating method at $100^{\circ} \mathrm{C}$ for 10 min with $0.01 \mathrm{M}$ citrate buffer ( $\mathrm{pH}$ 6.0). Endogenous peroxidase activity was quenched with methanol-hydrogen peroxide (3\%) for 
15 min. After blocking with unrelated antiserum, the slides were incubated with the $\mathrm{UbcH} 10$ primary monoclonal antibody (Boston Biochem, Cambridge, MA, USA) at 1:200 dilution and incubated overnight at $4^{\circ} \mathrm{C}$. After washing with Tris-buffered saline and Tween-20 (TBST), the sections were incubated with secondary antibodies followed by peroxidase-labeled streptavidin (Dako, Carpinteria, CA, USA) for $20 \mathrm{~min}$ at room temperature, and visualized with 3,3'-diaminobenzidine and counterstained with hematoxylin. Unrelated IgG was used instead of the primary antibody as a negative control.

\section{Results}

UbcH10 expression in 12 gastric carcinoma cell lines. To investigate the role of $\mathrm{UbcH} 10$ in gastric cancer, 12 gastric cancer cell lines (SGC-7901, AGS, NCI-N87, HS-746T, MKN-45, KATO III, NCI-SNU-1, SNU-5, SNU-16, GAXC023, GAXC031 and GAXC066) were examined to determine the UbcH10 expression levels by western blot analyses. Among these cell lines, GAXC023, GAXC03 and GAXC066 are primary tumor cell lines established recently in our laboratory from gastric cancer PDX models (30).

Diverse UbcH10 expression levels were observed in these 12 cell lines, with relative high expression levels observed in KATO III, SGC-7901, GAXC-023 and GAXC-031 cells, and much lower expression levels noted in NCI-N87, HS-746T and NCI-SNU-1 cells (Fig. 1A and B).

Knockdown of UbcH10 expression in gastric tumor cells inhibits proliferation. SGC-7901 and KATO III, both of which express $\mathrm{UbcH} 10$ at a high level, were selected for the RNA interference study. siRNA transfection led to 90 and $98 \%$ inhibition of UbcH10 expression in the SGC-7901 and KATO III cells, respectively (Fig. 2A).

Then, MTT cell proliferation assay was used to test the effect of UbcH10 knockdown. Cell growth was significantly slower in the UbcH10-knockdown SGC-7901 and KATO III cells when compared with those transfected with scramble RNAs (Fig. 2B).

Flow cytometric analysis with PI staining was then used to study cell cycle distribution. UbcH10-knockdown SGC-7901 cells displayed markedly reduced percentages of $\mathrm{S}$ and G2/M phase populations (Fig. 2C).

Inhibition of UbcH10 expression reduces cell fitness. Cell proliferation can be induced by changes in signaling pathways. Cells with or without UbcH10 knockdown were analyzed using western blot analyses. The cells with siRNA transfection were seeded in DMEM without serum overnight. Serum (1\%) was then used to stimulate cell signaling.

Phospho-ERK, phospho-Akt and phospho-p38 were attenuated with UbcH10 knockdown in the SGC-7901 cells (Fig. 3A and B). Similar changes were also observed in the same experiment using KATO III cells (data not shown).

The decrease in cell signaling and proliferation may be a sign of reduced cell fitness. To investigate this, the role of UbcH10 was then studied in cisplatin $(10 \mu \mathrm{M})$-induced gastric tumor cell apoptosis with flow cytometry using FITC-Annexin V and PI staining. Knockdown of UbcH10 in these 2 gastric cancer

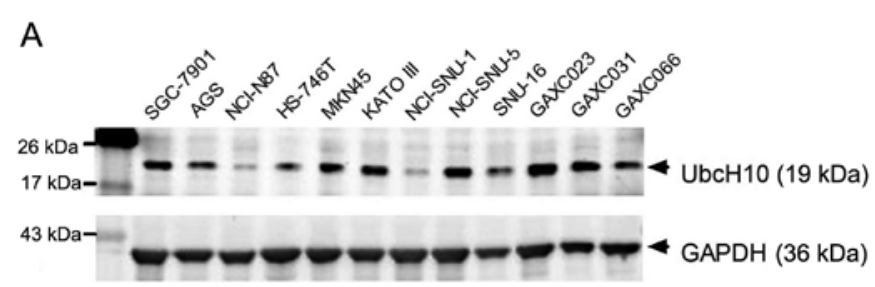

$\mathrm{B}$

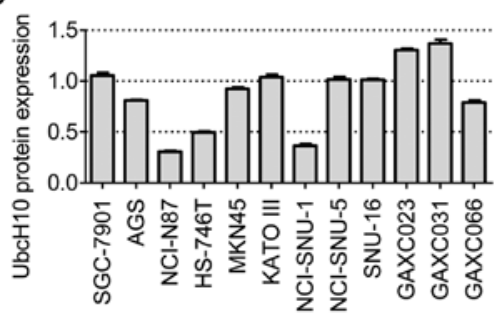

Figure 1. Expression of UbcH10 in 12 gastric carcinoma cell lines. (A) Cell lysates were prepared and analyzed for UbcH10 expression by western blotting. (B) Quantification of UbcH10 protein expression level in 12 cell lines. The ratio of UbcH10 to GAPDH was quantified using software ImageJ and normalized against SGC-7901 which was assigned the expression level of 1.0. Results are representative of 3 independent experiments. Columns, mean $(\mathrm{n}=3)$; bars, mean $\pm \mathrm{SD}$.

cell lines resulted in increases in apoptosis and eventual death following exposure to cisplatin treatment (Fig. 3C).

The data suggest that knockdown of UbcH10 in gastric cancer cells reduced tumor cell proliferation, serum-induced signal transduction and increased susceptibility to cisplatin-induced cell death.

Overexpression of $\mathrm{UbcH} 10$ in gastric tumor cells promotes cell proliferation and inhibits apoptosis. To confirm these findings, we constructed the UbcH10 expression plasmid for the overexpression studies in the NCI-N87 and HS-746T cells, Two of the gastric cancer cell lines showed low UbcH10 expression (Fig. 1).

The western blot analysis showed the enhanced expression level of UbcH10 in the two cell lines after the transfection (Fig. 4A).

MTT assay was used to test the cell proliferation $24 \mathrm{~h}$ after transient transfection of UbcH10. In accordance with the previous gene knockdown data, the overexpression of UbcH10 promoted tumor cell growth (Fig. 4B).

The overexpression of UbcH10 also led to reduced cisplatin-induced cell apoptosis as detected by Hoechst staining and apoptotic nuclei quantification (Fig. 4C).

Immunohistochemical (IHC) analysis of $\mathrm{UBCH} 10$ protein expression in gastric carcinoma patient samples. The role of $\mathrm{UbcH} 10$ in gastric cancer was supported both by the UbcH10 overexpression and the knockdown studies. Next, clinical gastric cancer patient samples were examined to evaluate the clinical relevance of the findings.

The expression of $\mathrm{UbcH} 10$ was examined in 59 cases of confirmed cases of gastric cancers by IHC analysis. Most of the cancer tissues showed different levels of $\mathrm{UbcH} 10$ expression at the tumor area; 19 samples displayed clear positive staining 


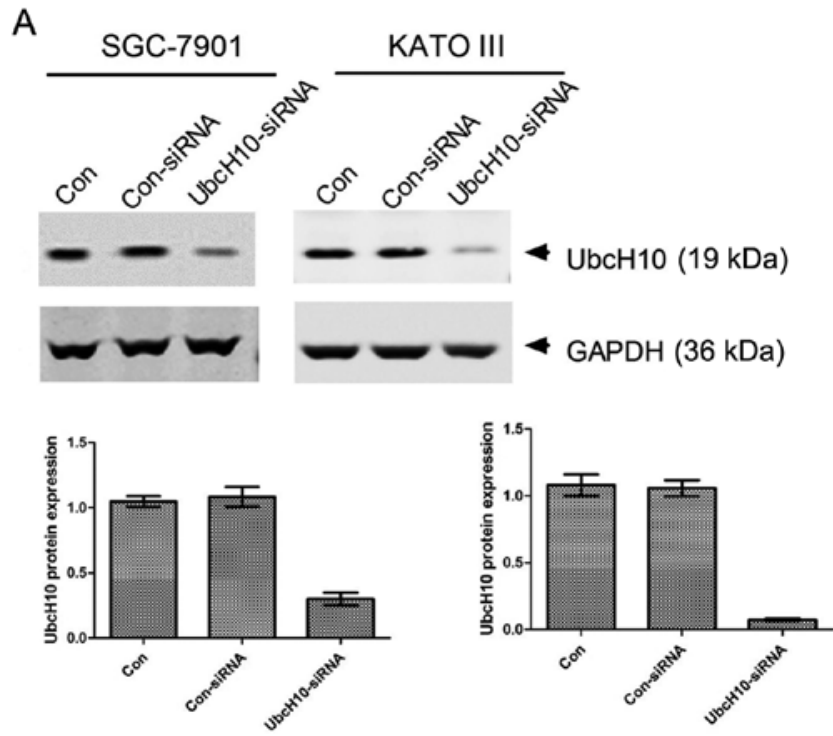

B

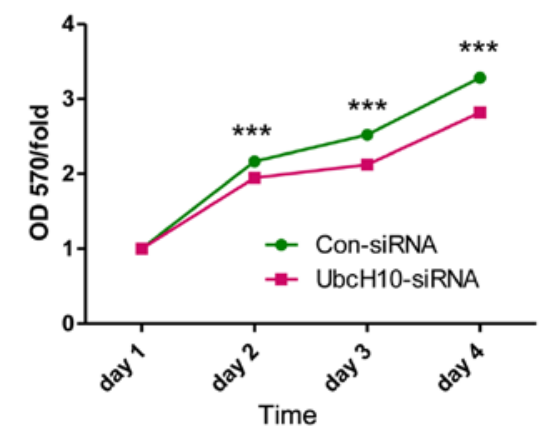

C

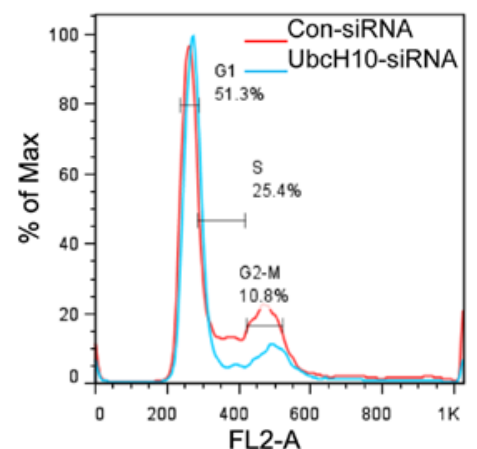

KATO III

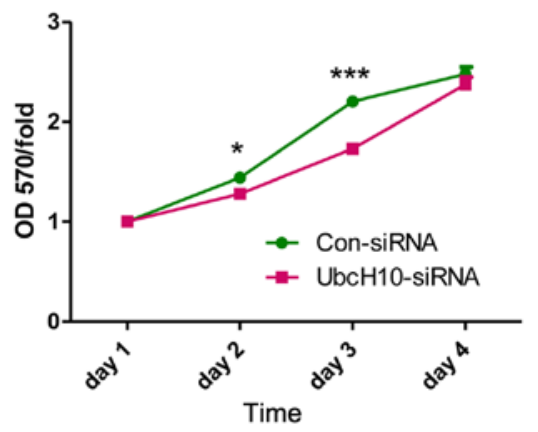

SGC-7901

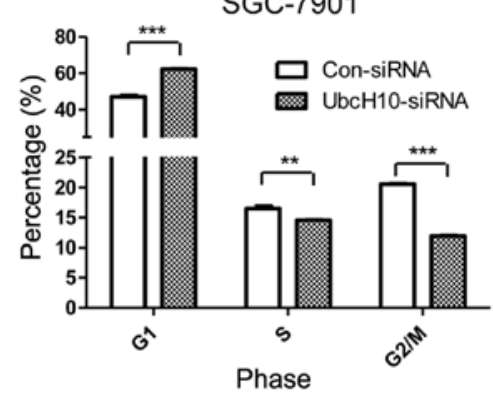

Figure 2. Knockdown of UbcH10 expression by siRNA inhibits cell proliferation and cell cycle progression. (A) Inhibition of UbcH10 expression in SGC-7901 and KATO III cells. Cells were transiently transfected with the scrambled or UbcH10-siRNA using Lipofectamine 2000. Con, untreated cells; Con-siRNA, scrambled siRNA; UbcH10-siRNA, UbcH10 targeted siRNA. Cell lysates were prepared and analyzed for the protein expression of UbcH10 by western blotting $36 \mathrm{~h}$ post-transfection. The UbcH10 expression level is presented in the lower panels. The ratio of UbcH10 to actin was quantified using software ImageJ. Columns, mean ( $\mathrm{n}=3$ ); bars, mean \pm SD. (B) SGC-7901 and KATO III cells were transfected with scramble or UbcH10 siRNA, respectively, and cell proliferation was analyzed by MTT assay in the subsequent 4 days. Values of optical density (OD) were obtained by the absorbance at the dual wavelengths $570 / 700 \mathrm{~nm}\left({ }^{*} \mathrm{P}<0.05,{ }^{* * *} \mathrm{P}<0.001\right.$; two-way ANOVA with Bonferronni post-tests). (C) SGC-7901 and KATO III cells were harvest in $70 \%$ ethanol $36 \mathrm{~h}$ after transfection with scramble or UbcH10-siRNA for cell cycle analyses by flow cytometry. Results were analyzed using FlowJo software. Bars, mean \pm SD $\left({ }^{* *} \mathrm{P}<0.01,{ }^{* * * *} \mathrm{P}<0.001\right.$; two-way ANOVA with Bonferronni post-tests).

(score of +), 23 samples displayed strong staining (score of ++ ) and another 7 samples exhibited very strong staining (score of +++) (Fig. 5). No signals were detected in the neighboring mesenchymal or IgG-negative control. Overall, 49 out of 59 cases $(83 \%)$ showed significant UbcH10 expression in the cancerous tissues when compared with that noted in the neighboring mesenchyma (Table I).

\section{Discussion}

As a critical regulator of cell cycle progression, $\mathrm{UbcH} 10$ is considered as a tumor-promoter gene in the conventional view among diverse cancers. As a member of the anaphase promoting complex or cyclosome (APC/C), UbcH10 not only regulates and controls the cell cycle (31), but also takes part in 
A
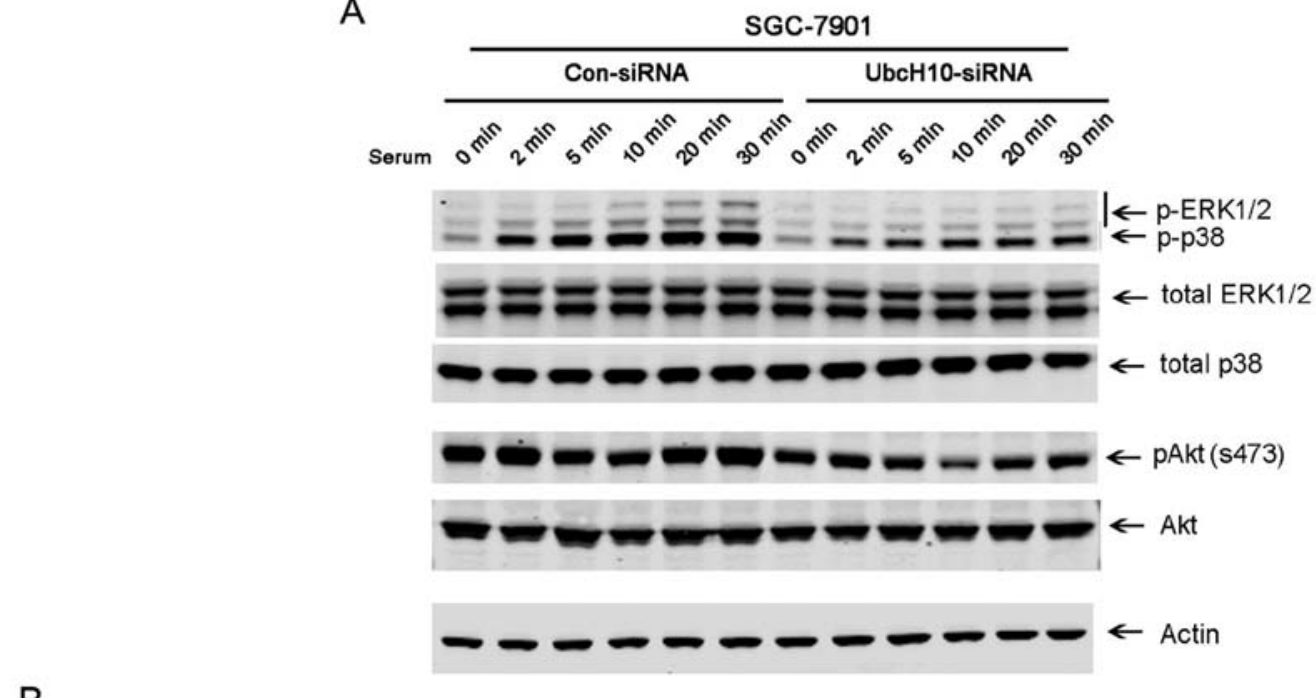

B
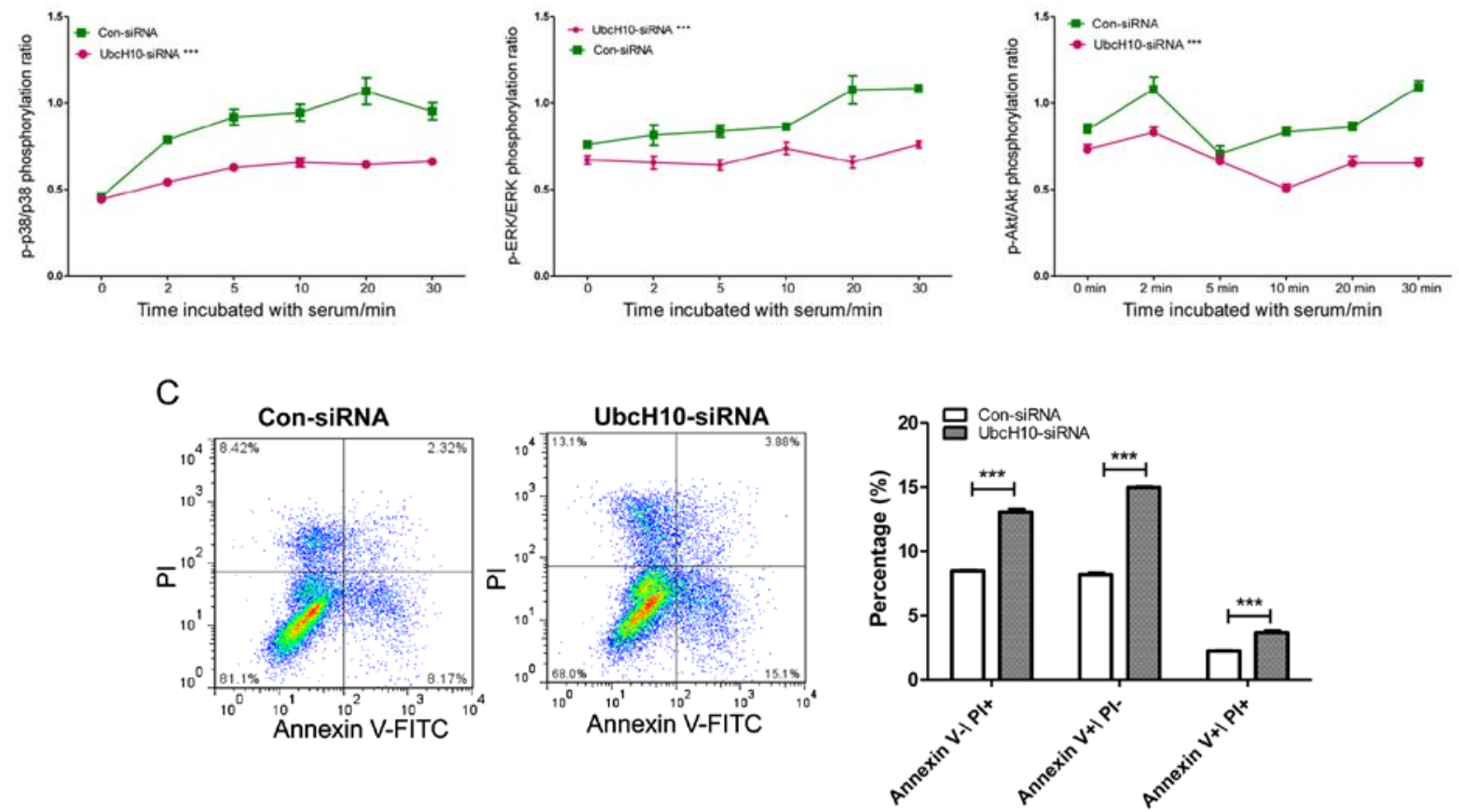

Figure 3. Signaling pathways and promotion of apoptosis. (A) SGC-7901 and KATO III cells were starved in serum-free medium overnight after transfection with siRNAs for $24 \mathrm{~h}$. Cells were harvested for western blot assay after stimulation with $1 \%$ serum and harvested at the time points indicated (B) Quantification of, p38, ERK and Akt phosphorylation in the SGC-7901 cells. Ratios of phosphorylated protein to total protein were analyzed with ImageJ software. ${ }^{* * * *}$ p $<0.0001$ compared to con-siRNA; two-tailed ANOVA. (C) KATO III cells were transfected with scramble or UbcH10-siRNA and cultured for $24 \mathrm{~h}$, and then were treated with $10 \mu \mathrm{M}$ cisplatin for $24 \mathrm{~h}$ before analyses of apoptosis/necrosis using flow cytometry. Results were analyzed using FlowJo software. ${ }^{* * *}$ p $<0.0001$ compared to con-siRNA; two-way ANOVA with Bonferronni post-tests.

initiation, progression and transformation (15) and significantly shows increased expression in HCC and other tumor tissues. There are no reports concerning the relevance of $\mathrm{UbcH} 10$ in gastric carcinoma. Thus, we utilized several gastric carcinoma cell lines for UbcH10 knockdown and overexpression studies.

In the present study, we found that inhibition of UbcH10 expression suppressed cell proliferation and altered the cell to become more sensitive to apoptosis accompanied by cell cycle change and cell survival signaling attenuation. In agreement with these data, the overexpression of UbcH10 promoted cell proliferation and resistance to cisplatin-induced apoptosis. UbcH10 was also overexpressed in 3 PDX-derived primary cell lines established in our laboratory. These cell lines had only limited passages in vitro and may be more closely related to current clinical diseases than traditional cancer cell lines.

More importantly, significant expression of UbcH10 protein was detected in patient tumor tissues, but not in adjacent mesenchyma among $83 \%$ of the gastric carcinoma patients (49 out of 59). In addition, with a limited data set, we also noted that the highest UbcH10 expression was also correlated with poorly differentiated cancer, suggesting that UbcH10 expression may be associated with poor prognosis. This issue may require future patient follow-up studies or retrospective analyses of a large volume of clinical data and 
A
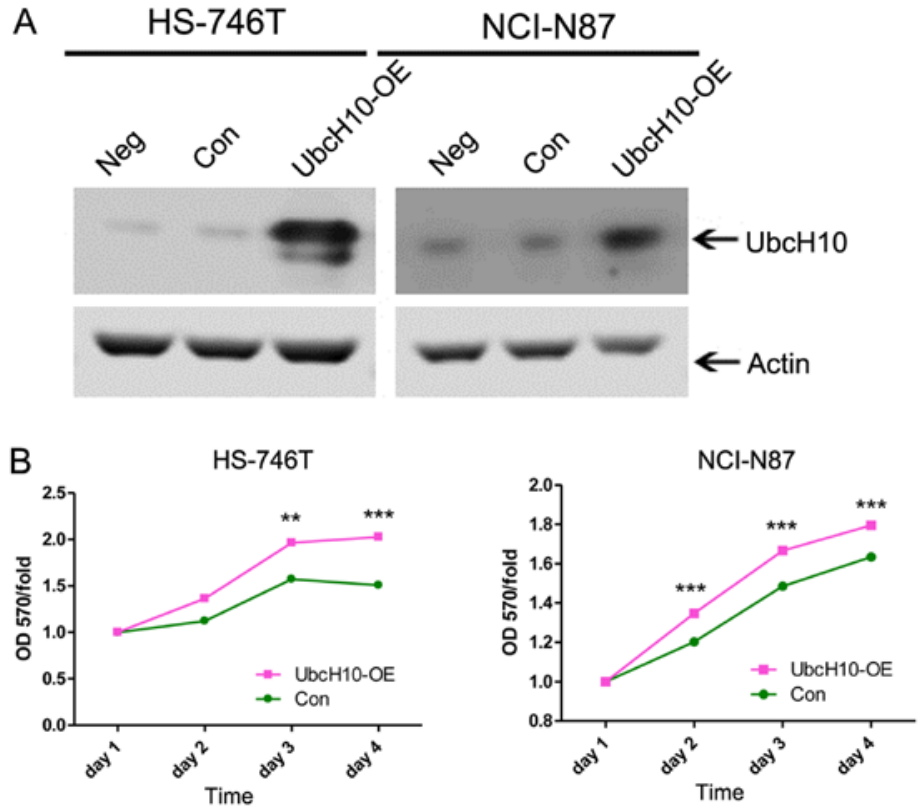

C

C
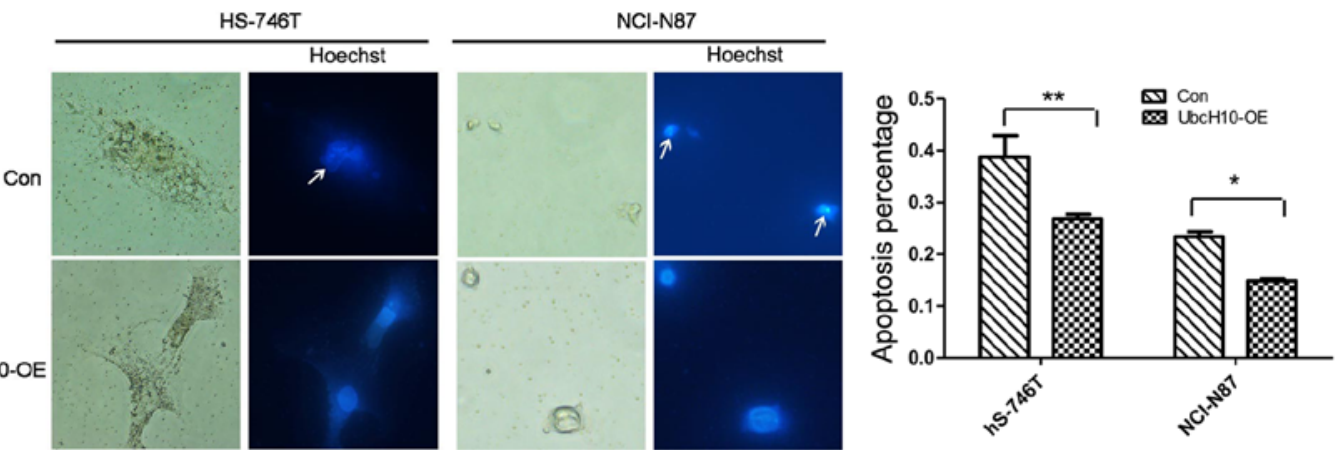

Figure 4. Overexpression of UbcH10 induced by plasmid transient transfection promotes cell proliferation, cell cycle and inhibits apoptosis. (A) FLVX-GFPIRES-UbcH10 was transiently transfected into HS-746T and NCI-N87 cells, respectively, as UbcH10-OE; cells treated with FLVX-GFP-IRES empty plasmid as well as untreated cells were used as negative controls. (B) HS-746T and NCI-N87 cells were transfected with different plasmids, and cell proliferation was analyzed by MTT assay in the subsequent 4 days. Values of optical density (OD) were obtained by the absorbance at the dual wavelengths $570 / 700 \mathrm{~nm}$. Bars, mean $\pm \mathrm{SD}\left({ }^{* *} \mathrm{P}<0.01,{ }^{* * *} \mathrm{P}<0.001\right.$; two-way ANOVA with Bonferroni post-tests). (C) HS-746T and NCI-N87 cells transfected with FLVX-GFP-IRES-UbcH10 or control plasmids were treated with $10 \mu \mathrm{M}$ cisplatin for $24 \mathrm{~h}$. Cells were fixed with $4 \%$ paraformaldehyde followed by Hoechst 33258 staining. The images were captured by fluorescence microscope, and quantification is presented in the right panels. Results are representative of 3 independent experiments. Columns, mean $(\mathrm{n}=3)$; bars, mean $\pm \mathrm{SD}\left({ }^{*} \mathrm{P}<0.05,{ }^{* *} \mathrm{P}<0.01\right.$; t-test).
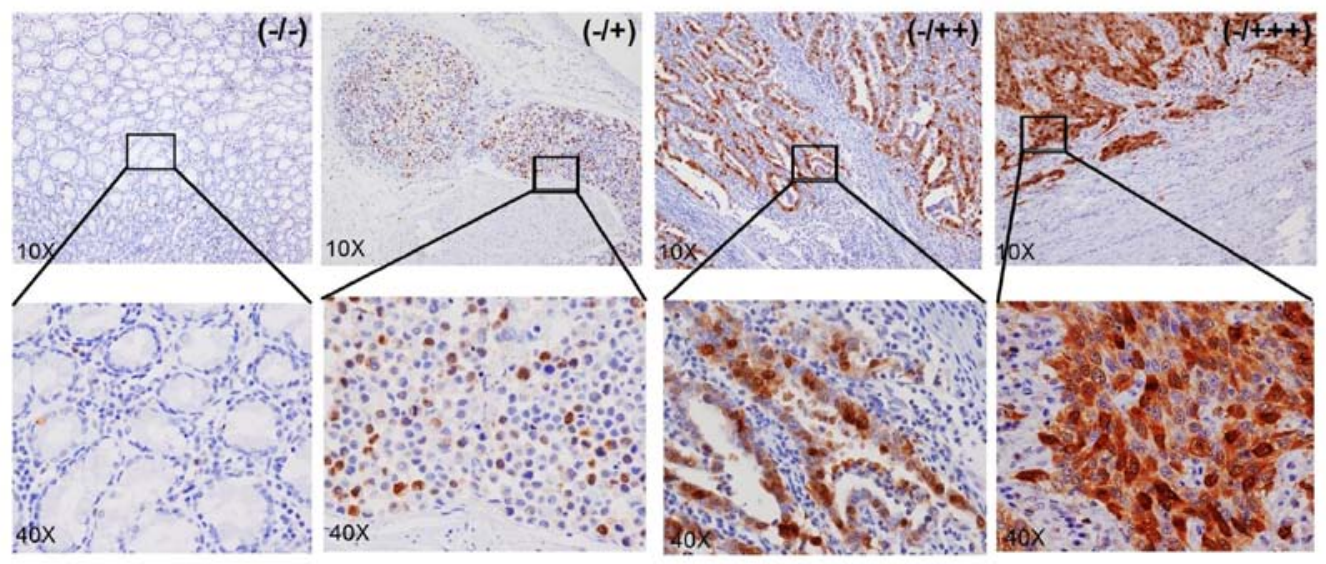

Figure 5. UbcH10 presents a high expression level in gastric carcinoma, but not in adjacent mesenchyma. Upper panels, magnification, x10; lower panels, magnification of $\mathrm{x} 40$ of the area shown in the boxes in the upper panels. Left panels, UbcH10 expression level was marked as (-/-) indicating negative for both mesenchyma and tumor area; second panels, UbcH10 was detect in cancer tissue (+) but not in normal mesenchyma tissue (-); third panels, strong UbcH10 expression was detected in cancer tissue (++) and normal mesenchyma tissue was negative (-); right panels, UbcH10 expression level was very strong in cancer tissue (+++) while normal mesenchyma tissues remained negative (-). 
Table I. UbcH10 expression in gastric cancer patient tumors and mesenchyme.

\begin{tabular}{|c|c|c|c|c|}
\hline $\begin{array}{l}\text { Staining } \\
\text { of tumors }\end{array}$ & $\begin{array}{l}\text { Staining of } \\
\text { neighboring } \\
\text { mesenchyme }\end{array}$ & $\begin{array}{c}\text { No. of } \\
\text { samples }\end{array}$ & $\begin{array}{c}\text { Percentage } \\
\text { of the } 59 \\
\text { samples }(\%)\end{array}$ & $\begin{array}{c}\text { Accumulative } \\
\%\end{array}$ \\
\hline+++ & - & 7 & 11.86 & 11.86 \\
\hline++ & - & 19 & 32.20 & 44.06 \\
\hline+ & - & 23 & 38.98 & 83.04 \\
\hline- & - & 10 & 16.95 & 100.00 \\
\hline
\end{tabular}

samples. If confirmed, Ubch10 expression level can serve as a useful biomarker for diagnosis and prognosis.

Amplifications of certain chromosomal regions have been identified in many types of cancers. The gene $U B E 2 C$ coding $\mathrm{UbcH} 10$ was reported to form genomic amplification which led to the increase in UBE2C expression in colon cancer, thyroid carcinoma and prostate cancer (32-34). A UBE2C genomic amplification may contribute to the overexpression of $\mathrm{UbcH} 10$ observed in at least some of the patient gastric cancer samples and gastric cancer cell lines. A systemic genomic analysis of the patient samples may offer a more definitive answer.

Notably, knockdown of UbcH10 expression in 2 overexpressing cell lines resulted in slightly different responses. In the SGC-7901 cells, such knockdown led to reduced percentages of S and G2M phase cells, while in the KATO III cells, G2/M phases were largely unaffected with changes predominantly in the $\mathrm{S}$ phases (data not shown). In addition, the changes in protein phosphorylation were more prominent in the UbcH10-knockdown KATO III cells in the absence of serum stimulation. These data suggest that the exact mechanism of how UbcH10 promotes tumor cell proliferation and resistance to apoptosis also depends on the 'wiring' of the cells, making detailed mechanistic studies more difficult.

Manipulating the ubiquitin pathways in cancer treatment has become one of the current focuses of research, despite that targeting these pathways has been largely premature in the clinic. The tumor promoting role of UbcH10 may make it a valuable therapeutic target.

Overall, our results indicated that overexpression of UbcH10 leads to increased cancer cell proliferation and reduced cancer cell apoptosis in gastric cancer cell lines, in agreement with previous findings in other types of cancers (20,35-38), further validated the tumor-promoting role of UbcH10. These data, and more importantly, its overexpression in gastric cancer patient samples while its absence in adjacent mesenchyma, suggest that UbCH10 may represent a potential biomarker for the diagnosis and prognosis or a potential therapeutic target in gastric carcinoma.

\section{Acknowledgements}

The present study was supported by the Shanghai Municipal Commission of Science and Technology (grant no. 14DZ2252000), and the Minhang Commission of Health Research (grant no. 2012MW16).

\section{References}

1. Ferlay J, Soerjomataram I, Dikshit R, Eser S, Mathers C, Rebelo M, Parkin DM, Forman D and Bray F: Cancer incidence and mortality worldwide: Sources, methods and major patterns in GLOBOCAN 2012. Int J Cancer 136: E359-E386, 2015.

2. Hartgrink HH, Jansen EP, van Grieken NC and van de Velde CJ: Gastric cancer. Lancet 374: 477-490, 2009.

3. Lin Y, Ueda J, Kikuchi S, Totsuka Y, Wei WQ, Qiao YL and Inoue M: Comparative epidemiology of gastric cancer between Japan and China. World J Gastroenterol 17: 4421-4428, 2011.

4. Sharma MR and Schilsky RL: GI cancers in 2010: New standards and a predictive biomarker for adjuvant therapy. Nat Rev Clin Oncol 8: 70-72, 2011.

5. Smyth EC and Cunningham D: Gastric cancer in 2012: Defining treatment standards and novel insights into disease biology. Nat Rev Clin Oncol 10: 73-74, 2013.

6. Nobili S, Bruno L, Landini I, Napoli C, Bechi P, Tonelli F, Rubio CA, Mini E and Nesi G: Genomic and genetic alterations influence the progression of gastric cancer. World $\mathrm{J}$ Gastroenterol 17: 290-299, 2011.

7. Jang BG and Kim WH: Molecular pathology of gastric carcinoma. Pathobiology 78: 302-310, 2011.

8. Yasui W, Sentani K, Sakamoto N, Anami K, Naito Y and Oue N: Molecular pathology of gastric cancer: Research and practice. Pathol Res Pract 207: 608-612, 2011.

9. Tan IB, Ng I, Tai WM and Tan P: Understanding the genetic basis of gastric cancer: Recent advances. Expert Rev Gastroenterol Hepatol 6: 335-341, 2012

10. A-Hassan E, Heinz WF, Antonik MD, D'Costa NP, Nageswaran S, Schoenenberger CA and Hoh JH: Relative microelastic mapping of living cells by atomic force microscopy. Biophys J 74: 1564-1578, 1998.

11. Pickart CM and Fushman D: Polyubiquitin chains: Polymeric protein signals. Curr Opin Chem Biol 8: 610-616, 2004.

12. Okamoto Y, Ozaki T, Miyazaki K, Aoyama M, Miyazaki M and Nakagawara A: UbcH10 is the cancer-related E2 ubiquitin-conjugating enzyme. Cancer Res 63: 4167-4173, 2003.

13. Rape M and Kirschner MW: Autonomous regulation of the anaphase-promoting complex couples mitosis to S-phase entry. Nature 432: 588-595, 2004.

14. Rape M, Reddy SK and Kirschner MW: The processivity of multiubiquitination by the APC determines the order of substrate degradation. Cell 124: 89-103, 2006.

15. van Ree JH, Jeganathan KB, Malureanu L and van Deursen JM: Overexpression of the E2 ubiquitin-conjugating enzyme UbcH10 causes chromosome missegregation and tumor formation. J Cell Biol 188: 83-100, 2010.

16. Perrotta I, Bruno L, Maltese L, Russo E, Donato A and Donato G: Immunohistochemical analysis of the ubiquitinconjugating enzyme UbcH10 in lung cancer: A useful tool for diagnosis and therapy. J Histochem Cytochem 60: 359-365, 2012.

17. Pallante P, Berlingieri MT, Troncone G, Kruhoffer M, Orntoft TF, Viglietto G, Caleo A, Migliaccio I, DecaussinPetrucci M, Santoro M, et al: UbcH10 overexpression may represent a marker of anaplastic thyroid carcinomas. Br J Cancer 93: 464-471, 2005.

18. Kefeli M, Yildiz L, Celik H, Tosun M and Karagoz F: UbcH10 expression in benign, hyperplastic, and malignant endometrial curetted materials: A tissue microarray study. Int J Surg Pathol 20: 360-366, 2012.

19. Shen Z, Jiang X, Zeng C, Zheng S, Luo B, Zeng Y, Ding R, Jiang H, He Q, Guo J, et al: High expression of ubiquitin-conjugating enzyme 2C (UBE2C) correlates with nasopharyngeal carcinoma progression. BMC Cancer 13: 192, 2013.

20. Ieta K, Ojima E, Tanaka F, Nakamura Y, Haraguchi N, Mimori K, Inoue $\mathrm{H}$, Kuwano $\mathrm{H}$ and Mori M: Identification of overexpressed genes in hepatocellular carcinoma, with special reference to ubiquitin-conjugating enzyme E2C gene expression. Int J Cancer 121: 33-38, 2007.

21. Berlingieri MT, Pallante P, Sboner A, Barbareschi M, Bianco M, Ferraro A, Mansueto G, Borbone E, Guerriero E, Troncone G, et al: UbcH10 is overexpressed in malignant breast carcinomas. Eur J Cancer 43: 2729-2735, 2007.

22. Fujita T, Ikeda H, Taira N, Hatoh S, Naito M and Doihara H: Overexpression of UbcH10 alternates the cell cycle profile and accelerate the tumor proliferation in colon cancer. BMC Cancer 9: 87, 2009. 
23. Chen S, Chen Y, Hu C, Jing H, Cao Y and Liu X: Association of clinicopathological features with UbcH10 expression in colorectal cancer. J Cancer Res Clin Oncol 136: 419-426, 2010.

24. Jiang L, Huang CG, Lu YC, Luo C, Hu GH, Liu HM, Chen JX and Han HX: Expression of ubiquitin-conjugating enzyme E2C/UbcH10 in astrocytic tumors. Brain Res 1201: 161-166, 2008.

25. Chen SM, Jiang CY, Wu JY, Liu B, Chen YJ, Hu CJ and Liu XX: RNA interference-mediated silencing of UBCH10 gene inhibits colorectal cancer cell growth in vitro and in vivo. Clin Exp Pharmacol Physiol 37: 525-529, 2010

26. Jiang L, Bao Y, Luo C, Hu G, Huang C, Ding X, Sun K and Lu Y: Knockdown of ubiquitin-conjugating enzyme E2C/UbcH10 expression by RNA interference inhibits glioma cell proliferation and enhances cell apoptosis in vitro. J Cancer Res Clin Oncol 136: 211-217, 2010

27. Lodhia KA, Hadley AM, Haluska P and Scott CL: Prioritizing therapeutic targets using patient-derived xenograft models. Biochim Biophys Acta 1855: 223-234, 2015.

28. Choi SY, Lin D, Gout PW, Collins CC, Xu Y and Wang Y: Lessons from patient-derived xenografts for better in vitro modeling of human cancer. Adv Drug Deliv Rev 79-80: 222-237, 2014.

29. John T, Kohler D, Pintilie M, Yanagawa N, Pham NA, Li M, Panchal D, Hui F, Meng F, Shepherd FA, et al: The ability to form primary tumor xenografts is predictive of increased risk of disease recurrence in early-stage non-small cell lung cancer. Clin Cancer Res 17: 134-141, 2011.

30. Sicklick JK, Leonard SY, Babicky ML, Tang CM, Mose ES, French RP, Jaquish DV, Hoh CK, Peterson M, Schwab R, et al: Generation of orthotopic patient-derived xenografts from gastrointestinal stromal tumor. J Transl Med 12: 41, 2014.

31. Lin Y, Hwang WC and Basavappa R: Structural and functional analysis of the human mitotic-specific ubiquitin-conjugating enzyme, UbcH10. J Biol Chem 277: 21913-21921, 2002.
32. Takahashi Y, Ishii Y, Nishida Y, Ikarashi M, Nagata T, Nakamura T, Yamamori S and Asai S: Detection of aberrations of ubiquitin-conjugating enzyme E2C gene (UBE2C) in advanced colon cancer with liver metastases by DNA microarray and two-color FISH. Cancer Genet Cytogenet 168: 30-35, 2006.

33. Lee JJ, Au AY, Foukakis T, Barbaro M, Kiss N, Clifton-Bligh R, Staaf J, Borg A, Delbridge L, Robinson BG, et al: Array-CGH identifies cyclin D1 and $U B C H 10$ amplicons in anaplastic thyroid carcinoma. Endocr Relat Cancer 15: 801-815, 2008.

34. Tzelepi V, Zhang J, Lu JF, Kleb B, Wu G, Wan X, Hoang A, Efstathiou E, Sircar K, Navone NM, et al: Modeling a lethal prostate cancer variant with small-cell carcinoma features. Clin Cancer Res 18: 666-677, 2012.

35. Donato G, Iofrida G, Lavano A, Volpentesta G, Signorelli F, Pallante PL, Berlingieri MT, Pierantoni MG, Palmieri D, Conforti F, et al: Analysis of UbcH10 expression represents a useful tool for the diagnosis and therapy of astrocytic tumors. Clin Neuropathol 27: 219-223, 2008.

36. Lin J, Raoof DA, Wang Z, Lin MY, Thomas DG, Greenson JK, Giordano TJ, Orringer MB, Chang AC, Beer DG, et al: Expression and effect of inhibition of the ubiquitin-conjugating enzyme E2C on esophageal adenocarcinoma. Neoplasia 8: 1062-1071, 2006.

37. Troncone G, Guerriero E, Pallante P, Berlingieri MT, Ferraro A, Del Vecchio L, Gorrese M, Mariotti E, Iaccarino A, Palmieri EA, et al: UbcH10 expression in human lymphomas. Histopathology 54: 731-740, 2009.

38. Vasiljevic A, Champier J, Figarella-Branger D, Wierinckx A, Jouvet A and Fèvre-Montange M: Molecular characterization of central neurocytomas: Potential markers for tumor typing and progression. Neuropathology 33: 149-161, 2013. 